\title{
Toward an Approach to Product Design Process Modeling and Conflict Resolution for Collaborative Virtual Environment
}

\author{
Pengfei Zeng ${ }^{1,2, *}$, Yongping $\mathrm{Hao}^{2}$, Weiping Shao ${ }^{1}$ and Ze Tao ${ }^{1}$ \\ ${ }^{I}$ School of Mechanical Engineering, Shenyang Ligong University, Shenyang, Liaoning, 110159, P.R. China \\ ${ }^{2} R \& D$ Center of CAD/CAM Technology, Shenyang Ligong University, Shenyang, Liaoning, 110159, P.R. China
}

\begin{abstract}
According to engineering and technical staffs' requirements in inter-multidisciplinary domains collaborative product design, design process modeling method and conflict resolution algorithm are both put forward. The actual needs are deeply analyzed for multidisciplinary collaborative product design. By means of a third-party data file conversion format-.3D, a collaborative design mode and its framework structure are respectively presented based on a product sharing view model. For the design schemes of multiple users' collaborative activities process, a conflict resolution algorithm of design requests is also implemented. Finally, based on the J2EE architecture, a lightweight virtual network environment is developed to support collaborative design process, and its application validation work is also discussed. The feasibility and effectiveness method and architecture proposed have been also testified reasonable.
\end{abstract}

Keywords: Collaborative product design, activity process modeling, conflict resolution, lightweight virtual environment, file format interchange.

\section{INTRODUCTION}

In the modern product development, design cooperation more and more is emphasized on enterprises internal or external. It can integrate some core competitiveness from various different fields' enterprises to achieve all-win. It is very important that collaborative design process is auto-matically integrated and heterogeneous data or information is seamlessly shared and interoperated. But, this point also increases higher risks to protect original design data and other related intellectual assets from cooperative companies.

How to quickly and efficiently build low-cost secure and lightweight collaborative virtual environment (CVE), to establish interdisciplinary collaborative design process and conflict resolution method, so as to support multidisciplinary collaborative design make decision, it must be currently solved difficult problems. Liu Chen et al. [1] described a customer-driven product design and evaluation method for collaborative design environment to help product designers to generate appropriate design concepts and evaluate design alternatives. Dongjing $\mathrm{He}$ et al. [2] proposed a method for complex product collaborative design based on cloud service to solve the problems of distributed collaboration, resources or information sharing, and knowledge reuse among design teams. The reference [3] presented a collaborative design framework architecture that allows the definition and verification of constraints and meta-rules to industry in view of the design chain, and proposed a reference model is used common modeling language to solve assist design conflict resolution strategy through synchronization and negotiation process. The reference [4] analyzed the problems of the Korean automotive parts problems regarding communication protocols, design chain interoperability and synchronization.

In this paper, the authors with the help of preliminary research foundation, a lightweight CVE is proposed based on a sharing view model (SVM) to meet with demands from multiples domains product design intents and information exchange and sharing across whole product life cycle process. And a kind of design conflict resolution solutions is established in collaborative process for CVE. Finally, the application verification is discussed.

\section{MULTIDISCIPLINARY PRODUCT DESIGN RE- QUIREMENT ANALYSIS}

It is the current trend to collaborative product design that cooperative enterprises accomplish design activities together over multiple discipline domains. Because of differences of discipline domains and hardware platforms and software systems, incompatibility of data formats among collaborative design members and others, these have seriously hampered multidisciplinary collaborative product design (MCPD). It is also difficult to achieve design information and knowledge sharing and interoperability across geographically distributed heterogeneous platforms $[5,6]$. With the global competition intensifies, enterprises must be used collaboration to achieve product development, this is only way to significantly reduce design costs. However, it will also inevitable generate 


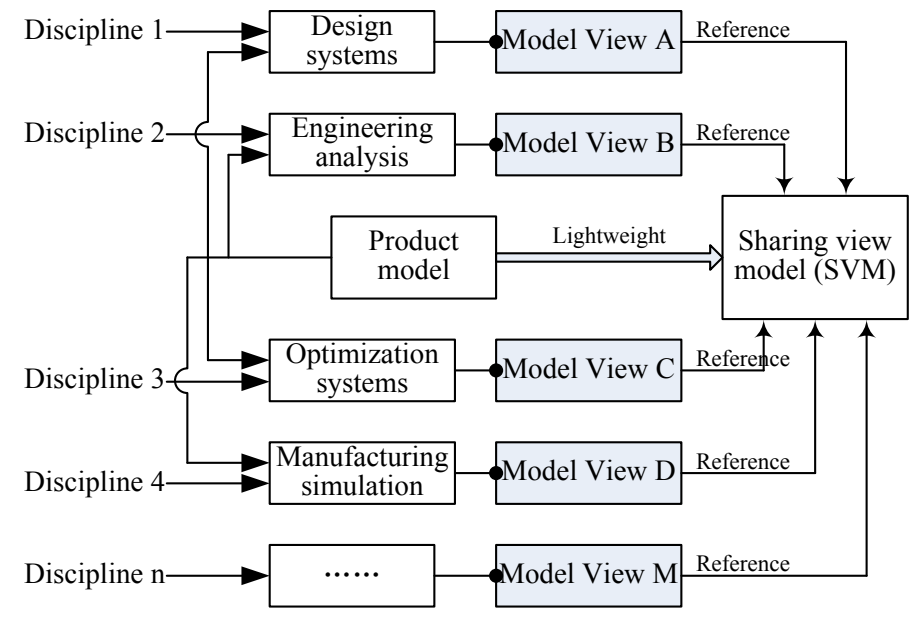

Fig. (1). Model Views Integration for Multiple Disciplines domains.

inter-change and sharing of product model and design information in the collaborative process. Therefore, we need a CVE to support effective design activities in the whole product life cycle process, and to complete data exchange and knowledge communication across enterprises and multidisciplinary domain engineering and technical staffs. The model data and design information generated or created in product design phase own different requirements and expression forms in other life cycles phases, such as in the manufacturing phase, purchasing phase, processing phase, logistics phase, technical support, sales, service and training, et al., and mutual collaboration and feedback of process requirements are essential in the different fields, of course, information exchange and data sharing is also frequently involved in the supply chain stages.

Under the current market environment, enterprise may be use different CAD/CAE software systems or applications. These are usually developed using different storage formats and program languages. In addition, when enterprise transits design data directly to others cooperation process, it may be cause two serious problems: firstly, data volume of design model transmitted is very large, and second, design knowledge and intelligence assets can easily be lost in product data and information from cooperative enterprises. So in the collaborative process, these problems arise, such as multi-source design applications, intellectual asset protection and data format conversion, and other new issues. Therefore, we need a kind of technology to achieve design models sharing and information seamless integration, to construct a lightweight virtual design space to support different domains cooperation between product design and manufacturing engineering. It can be able to some different locations discipline engineering technical staffs rely on the shared environment to accomplish product development. It is safe, convenient and flexible.

\section{SVM GENERATION AND PUBLISHING IN MCPD}

\subsection{Generation Method of Product SVM}

In order to information exchange and design feedback across the collaborative process, the product model is shown different model views (MV) in different engineering systems environment from multidisciplinary domains, as been shown in Fig. (1). We assume discipline 1 produces the MV A of the product model with a product design system, discipline 2 creates the MV B of the product model using engineering analysis system, discipline 3 obtains the MV C of the product model through engineering optimization system, discipline 4 establishes the relative MV D of the product model via manufacturing simulations, discipline $\mathrm{n}$ constructs the MV M of the product model by other engineering disciplines systems, and so on other MVs are related to systems design activities. Therefore, product model must implements design communication and semantic information sharing over different disciplines in collaborative process. Due to the differences existence of using application systems and software tools among multiple domains, so that the same product model is reflected to different semantic information expression in interdisciplinary collaboration process. In other words, in the collaborative product design environment, if a certain discipline designer wants to check other discipline views associated product model, he must have equipped with or installed appropriate discipline applications system. These make design costs greatly increase, will bring some security issues. The conversion of product model view among multiple disciplines will result missing and ambiguity on design information, data and engineering semantics. In addition, the inconsistent to understanding different MVs among interdisciplinary domain engineering designer, will also result many complex issues.

Moreover, with the increase of disciplines number involved, the interaction times will result in a geometric growth between different disciplines. Conversion between MVs and without order communication will take up a lot of human resources, network bandwidth and resources, it is time-consuming and inefficient, and will seriously hinder the effective realization of collaborative activities. If we can change the traditional product models exchange way, establishing a sharable product view model and application environment across multidisciplinary fields, make respective discipline fields to sharing a unified product MV. So that collaboration efficiency will be greatly improved, and costs will also be reduced overhead. As been shown in Fig. (1), N 
discipline fields share the same product MV to implement collaborative design activities, so the interaction patterns is greatly simplified and the distance has also been shortened among various discipline numbers, it is better beneficial to overall sequential distribution of product design information and global optimization of design achievements. In fact, these also achieve global optimization on the basis of solving a single discipline problem in the product design process. As a result of all-win solution has also generated.

\subsection{SVM Generation and Publishing Based-on the Third-Party .3D Format}

With the help of an extensible design visualization and collaboration work solutions-digital design communication platform provided by Actify Inc. [7], conversion and publishing schemes of product information SVM is achieved. The provided tool system allows $2 \mathrm{D}$ and $3 \mathrm{D}$ product data to seamless communication and integration for product manufacturing and global supply chains. Via providing many appropriate components and secondary development, CAD models with different file formats can be able to publish into a unified .3D format file. The storage, management, access control, and enterprise internal and external sharing of intermediate format document are realized through relative networks. The Actify can achieve security, confidentiality, and simple .3D file formats to design collaboration and supply chain cooperation in the case of without installing any native CAD design software. Users can use their product suites and 3D workspace functions to organize and publish relative product design drawing and document files, and can also generate 3D drawing from variety original CAD model file, and can also add design information and other relative documents, links, multiple models, marking and labeling, etc. to the model, and can employ it to protect the original CAD drawing document, meanwhile recipient does not need to install the relevant CAD systems or tools as well. Through the sharing design models, communication and exchange of $\mathrm{CAD}$ drawings and related information will be simplified.

Relative components are adopted as middleware tools to meet the needs of creating and publishing the product SVM for multidisciplinary field collaborative process using Actify, so that product model design finished can be converted into .3D format files. In the collaborative process, the original design data is submitted and on-demand checked out through a product data management system when designer completes design task. After the design data is submitted, through setting the address of source files model library of product design data, the address of destination file model library, making rules, and properties and trigger mechanism of format conversion functions, we can achieve timely generate product SVM needed in the collaborative design process, can record and manage related information the whole model conversion process produced via a database system. After the SVM is generated, its information will be published in the collaborative network. When collaborative participants login into the CVE system, he can check out or verify the appropriate model information through setting release mechanism of the SVM.

\section{COLLABORATIVE DESIGN PROCESS MODEL- ING AND FRAMEWORK DESIGN}

\subsection{Design Process Modeling Based on the SVM}

Establishing to collaborative design framework and its application sharing scheme, is a necessary condition to achieve lightweight security collaboration environment. In the collaborative process, due to some close correlation elements exist in activities, processes, data, management and information, the coupled relationship of design activities chain must be clearly established to manage and control the design process. In this paper, an extensible event-driven chain diagram (eEPC) model is put forward based on SVM of product information in the collaborative design process, as been shown in Fig. (2). In the Fig. (2), the CAD drawing library stores product design drawing and document, the sharing model library stores lightweight sharing model data, and the design information repository stores design information and related data.

When product designer completes assigned design task, he may submit and store design data and models to the CAD drawing library in collaborative design management system. The design models are converted to $.3 \mathrm{D}$ sharing model files that stored in sharing model library by a specific trigger conditions, meanwhile, the information of SVM released is published. In a certain collaborative process, user can ondemand participates in relevant collaborative activities across whole life cycle process. Other product engineers can browser, view, check, and mark up the model. Disciplinary designers may also join in the collaborative design through computer browser windows, in the collaborative process, design messages are stored in the design information repository, and information of the SVM will be also updated. When subsequent designer joins in the collaborative process, he can extract sharing model and product design information to complete collaborative design or model view. Finally, the design results are feedback to product designers to modify its design documents, or go ahead further collaborative process until a satisfactory feasible optimal solution is acquired. This process model is applied for synchronous real-time and asynchronous non-real-time collaborative design process. The storage pattern separating from model information to design information improves the response speed and cooperative efficiency of collaborative design event.

\subsection{Collaborative Design Framework Infrastructure}

Through the establishment of SVM, many collaborative participants, at the same time, in different locations, can join in the virtual workspace environment to carry on collaborative product design, and any participant can quickly get feedback information of design data from other collaborators. So that a more tightly integrated collaborative work mode is established. This collaborative model needs relatively higher network requirements to ensure design information to real time transmit and forward. For this purpose, a collaborative design framework is established in the CVE based on the SVM, as shown in Fig. (3). Through a kind of collaborative design interface (CDI), collaborator can access the CVE 


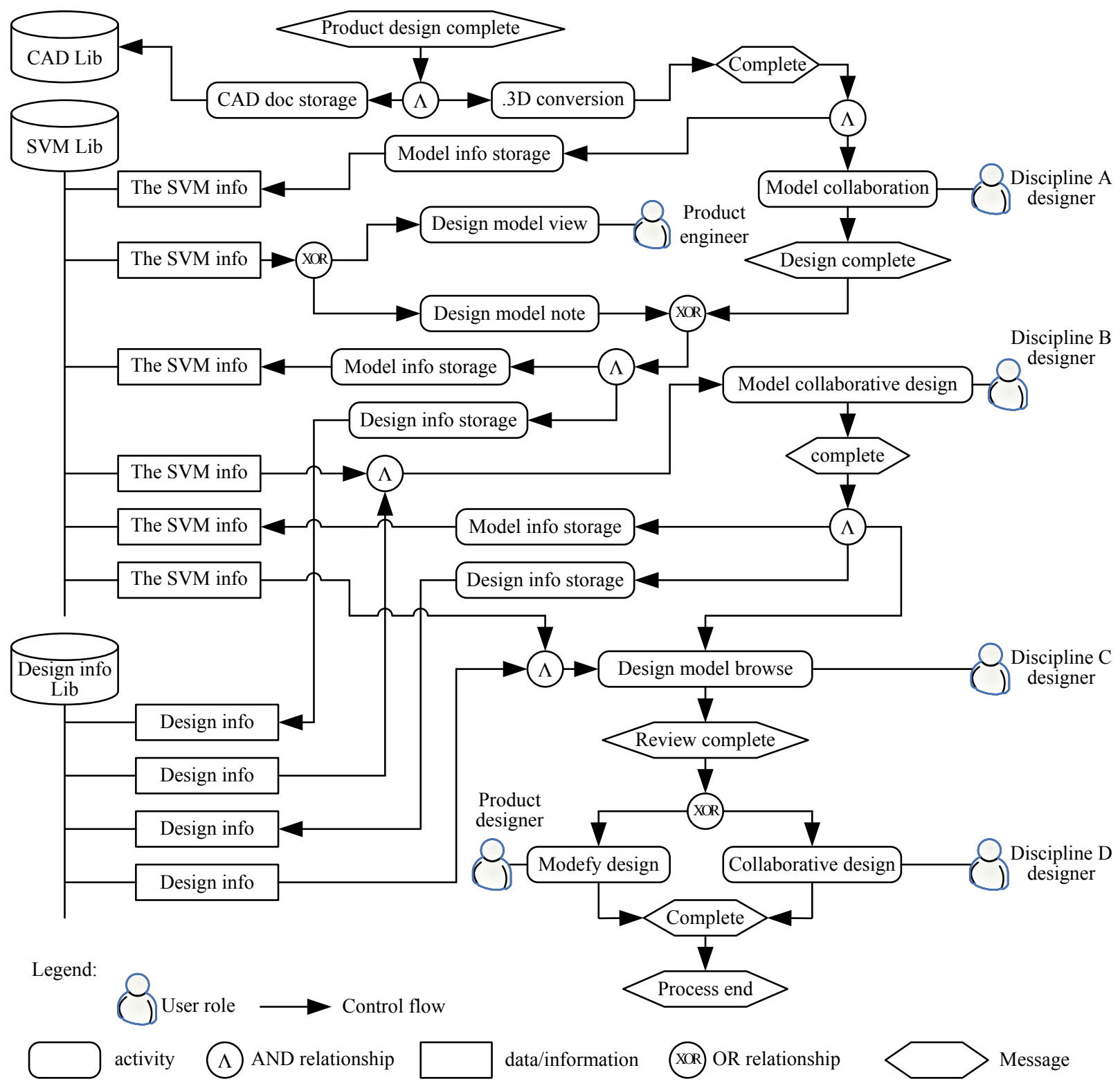

Fig. (2). Collaborative Product Design Process Modeling for SVM.

and carry out collaborative design activities based on the SVM generated and published, as well as preserve relevant design data and information the activities process generated. The CVE mainly includes two controller modules: the collaborative controller (CC) and the model convertion controller $(\mathrm{MCC})$.

There is a model controller (MC) consist in designers' browser side. The $\mathrm{MC}$ is responsible for send and receive message data, access control to the SVM. The CC is located in the collaborative design system server side, is responsible for conflict arbitration, model transformation management and strategies management in whole collaborative process. Through the CDI, collaborative designer can manipulate the SVM, can also implement access control by the MC, and can also send design message to collaborative server side by collaborative communication interface (CCI). When the collaborative design server receives design information, the system server real time forwards it to others collaborative users' browser side. When collaborative users' browser side receives design information transmitted, the updated SVM will be displayed in the user' browser window by means of the $\mathrm{MC}$ and the $\mathrm{CDI}$. In the server side, the $\mathrm{CC}$ converts a CAD design model to the SVM through a model convert interface (MCI), and receives and forwards design information and real-time immediate conflict arbitration through the CCI.

With the help of the SVM, network collaborative activity process implementation is not restricted with geographically location where collaboration participants sited in. On this account relevant design information only are transmitted among different discipline designers, so that network traffic pressure is reduced, and commercial security of detailed 


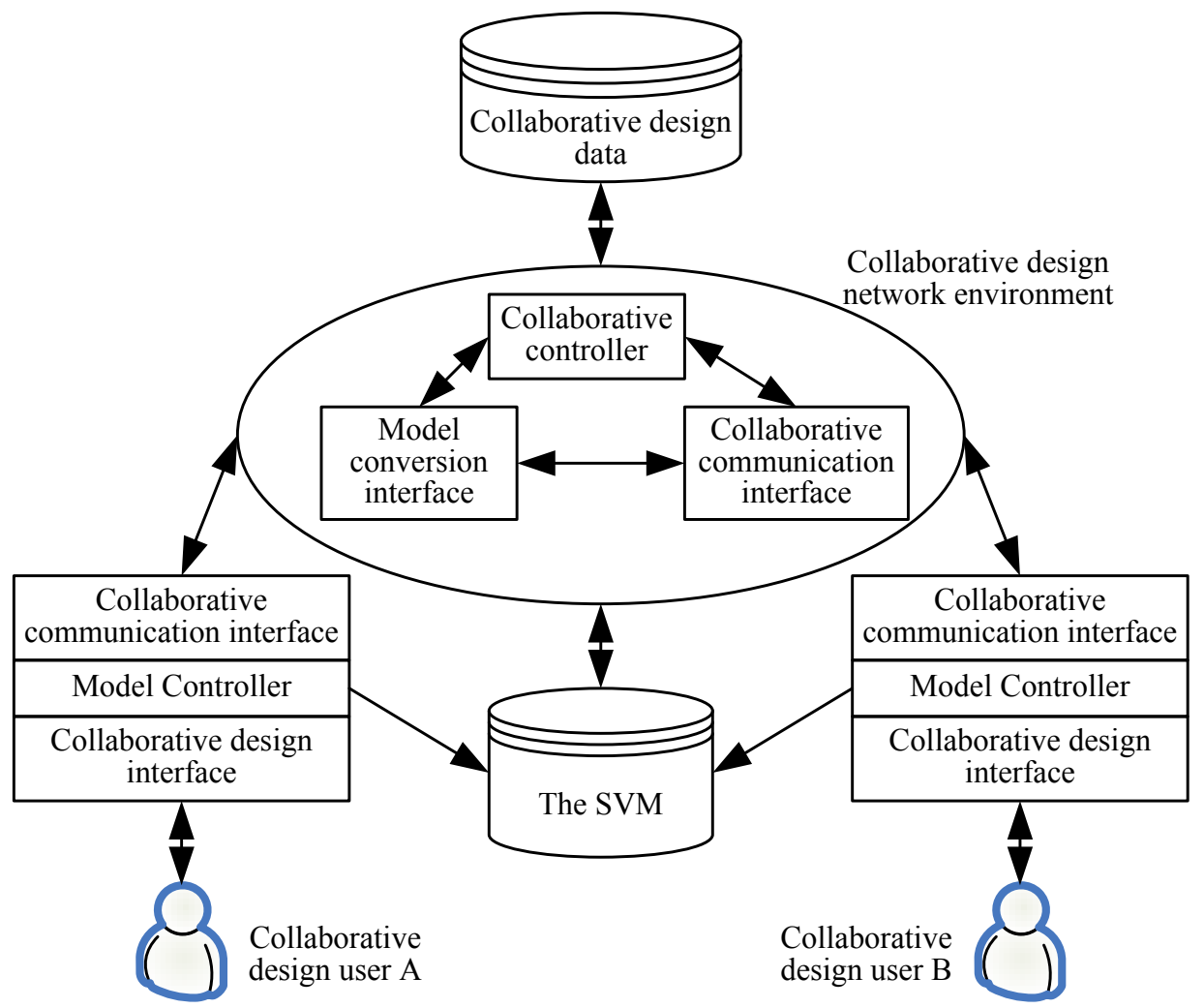

Fig. (3). Collaborative product design framework infrastructure.

design data is also protected from cooperative enterprises. Around the whole collaborative design management system, the framework and function modules design puts more emphasis on supporting distributed heterogeneous multi-level design groups coupling to collaborative process, to support synchronous and asynchronous collaborative work mode based on the Web browser in the network environment, providing the ability to session and information management of collaborative process, as well as gaining the ability to effectively control cooperation process.

\section{CONFLICT MODELING AND RESOLUTION FOR DESIGN REQUEST}

\subsection{Modeling of Design Conflict in Collaborative Process}

In order to meet the requirements of multidisciplinary synchronous collaborative process engineering and technical staffs participated in, the author describes an interactive control mechanism on a product sharing information model with considering design priority ranking level in the our previous works [8]. Therefore, while realizing collaborative design process based on the SVM, it is essential to take into account modeling design conflicts and providing resolution approach to prevent it occurrence, deadlock comparison of design token holding as well as competition abnormal phenomena in the design request queues. Moreover, for the special case of design priority comparison, some specific rules have also been set up, that is, when the collaborative design management system is dealing with design requests from a number of collaborative participants, subsequent design request from other collaborative users join in the design request queues is no longer processed.

For design request dealing with from many collaborative users, how select collaborative participants to allocating design token in fixed queue, and in essence it is a process of decision-making choice. From the control method of design interactive process, we can comprehensive consider the sequence of giving collaborative participants design token, on the mainly basis of design priority, sorting time, system role, status of network nodes located in and so on, to avoid conflict generation on the design resources and token. Thus, a waiting queue is defined to implement design requests for collaborative user $U$ in the CVE, as shown in follows:

$$
\begin{aligned}
& C o D_{-} Q=\left\{W_{Q 1},{ }^{W}{ }_{Q 2}, W_{Q 3}, \cdots \cdots, W_{Q n}\right\} \\
& W_{Q i}(U)=<P_{U}, R_{U}, Q_{U}, L_{U}>i=1,2, \cdots \cdots, n
\end{aligned}
$$

Where $P_{U}$ is defined as the collaborative users design priorities, $P_{U}=[0,1,2,3,4,5]$, the value of $P_{U}$ from 0 to 5 represents the design priority levels from a certain collaborative participant, if $P_{U}=0$ so indicate that the collaborative user design priority is lowest, and $P_{U}=5$ represents design priority is highest;

$R_{U}$ is defined as a collaborative users role, $R_{U}=[0,1,2$, 3 , 4], designer, disciplinary designer, system manager, 
project manager and chief designer are all roles consist in the collaborative system, $R_{U}$ values from 0 to 4 represent the assigned role value of collaborative participants;

$Q_{U}$ expresses the waiting or ranking time from collaborative user sends design request to be accepted, its value lists in seconds;

$L_{U}$ shows the status of network node collaborative user located in, $L_{U}=[0,1,2,3,4,5,6,7,8,9]$. The size of the $L_{U}$ value reflects the user's synthesis network state, for example, bandwidth of node, delay, jitter, loss rate and processing capabilities. For each collaborative user, we can comprehensive calculate its value ranges, and then assign a given $L_{U}$ value. $L_{U}=9$ shows the network node state is best where the collaborative user situates. If $L_{U}=0$, then the collaborative user have been unable to properly connect to the collaborative system or have already quit.

And, a ranking result of collaborative user $U$ in the design request queue expressed as:

$$
W_{Q \rightarrow U}=\left\{\begin{array}{l}
\omega_{1} \cdot P_{U}+\omega_{2} \cdot R_{U}+\omega_{3} \cdot Q_{U}+\omega_{4} \cdot L_{U} \quad L_{U} \neq 0 \\
0 \quad L_{U}=0 \\
\omega_{1}+\omega_{2}+\omega_{3}+\omega_{4}=1
\end{array}\right.
$$

Where, $\omega 1, \omega 2, \omega 3$, and $\omega 4$ respectively represent each factor weights. Depending on the size sorting weights calculated, the priorities that collaborative user $U$ implement synchronized design can be determined within the request queue. Assuming that the number collaborative users issuing design request is $N$, then, in a request processing time period, the sort waiting status about $N$ collaborative users can be obtained:

$$
M_{Q \rightarrow U / n}=\left[\begin{array}{cccc}
p_{1} & r_{1} & q_{1} & l_{1} \\
p_{2} & r_{2} & q_{2} & l_{2} \\
p_{3} & r_{3} & q_{3} & l_{3} \\
\cdot & \cdot & \cdot & \cdot \\
\cdot & \cdot & \cdot & \cdot \\
p_{n} & r_{n} & q_{n} & l_{n}
\end{array}\right] \times\left[\begin{array}{llll}
\omega_{1} & \omega_{2} & \omega_{3} & \omega_{4}
\end{array}\right]^{T}=\left[\begin{array}{c}
w_{U 1} \\
w_{U 2} \\
w_{U 3} \\
\cdot \\
\cdot \\
w_{U n}
\end{array}\right]
$$

Through calculating and comparing the size of value with $w_{U 1}, w_{U 2}, w_{U 3}, \ldots \ldots, w_{U n}$, the priority order the $N$ collaborative users implement design activities can be arranged out. Since the target and hierarchy structure are relatively simple, so the right weight value of index $\omega_{1}, \omega_{2}, \omega_{3}$, and $\omega_{4}$ can be directly determined by way of the analytic hierarchy process (AHP) [9] in actual operation.

\subsection{Design Conflict Resolve Algorithm}

In the development and implementation process of the collaborative design management system, based on expert scoring as well as a series of detailed steps and process of the AHP method, we have calculated and determined a set of weight values on each factor to meet the requirements of design request sorting, and also completed the necessary consistency test, they are $\omega_{1}=0.48, \omega_{2}=0.18, \omega_{3}=0.29$, $\omega_{4}=0.05$, respectively. Thus, a conflict resolution method to design request is obtained for multidisciplinary field users in collaborative process, as follows:

$$
\begin{aligned}
& \text { CreateDesignQueue(UserList) } \\
& \text { Begin } \\
& \text { set time_interval; } \\
& \text { initial user_list_array[][4]; } \\
& \text { while } \mathrm{t}<\text { time_interval do } \\
& \mathrm{i}=0 ;
\end{aligned}
$$

if received user_request

get $\mathrm{W}_{\mathrm{Q}}$ [user];

if $\mathrm{W}_{\mathrm{Q}}[$ user] $[3]=0$

delete the user;

end if

else if

add user_info into user_list_array[i][4];

$\mathrm{i}++$;

end if

end if

end while

for $\mathrm{j}=1: \mathrm{i}$;

count $\mathrm{w}_{\mathrm{U}(\mathrm{j})}=\mathrm{W}_{\mathrm{Q}} \times \omega$;

end for

compare $\mathrm{w}_{\mathrm{U}}$;

generate design_order();

return user_ranking_list;

End

With this algorithm, the design requests can be batched within a certain time intervals, the priority order of collaborative users design requests will be gotten within the collaborative group, thus design conflicts resolution is also achieved. Both to prevent invalid user access and to avoid over-long waiting time, in the collaborative design system, thus the system processing capacity and efficiency are ensured in a certain range, the system adaptability is also improved.

\section{APPLICATION CASE}

The authors have made some basic works to build on the model sharing method and virtual space environments [10]. In this section, we focus on further expansion and improvement the collaborative design management system developed, to implement applications and verifications system algorithms presented. The collaborative system is established integration with the J2EE architecture, using the browser/server mode to response design and service request, adopting combine Apache with Tomcat treatment as the Web server, its programming languages includes Java, JSP, 


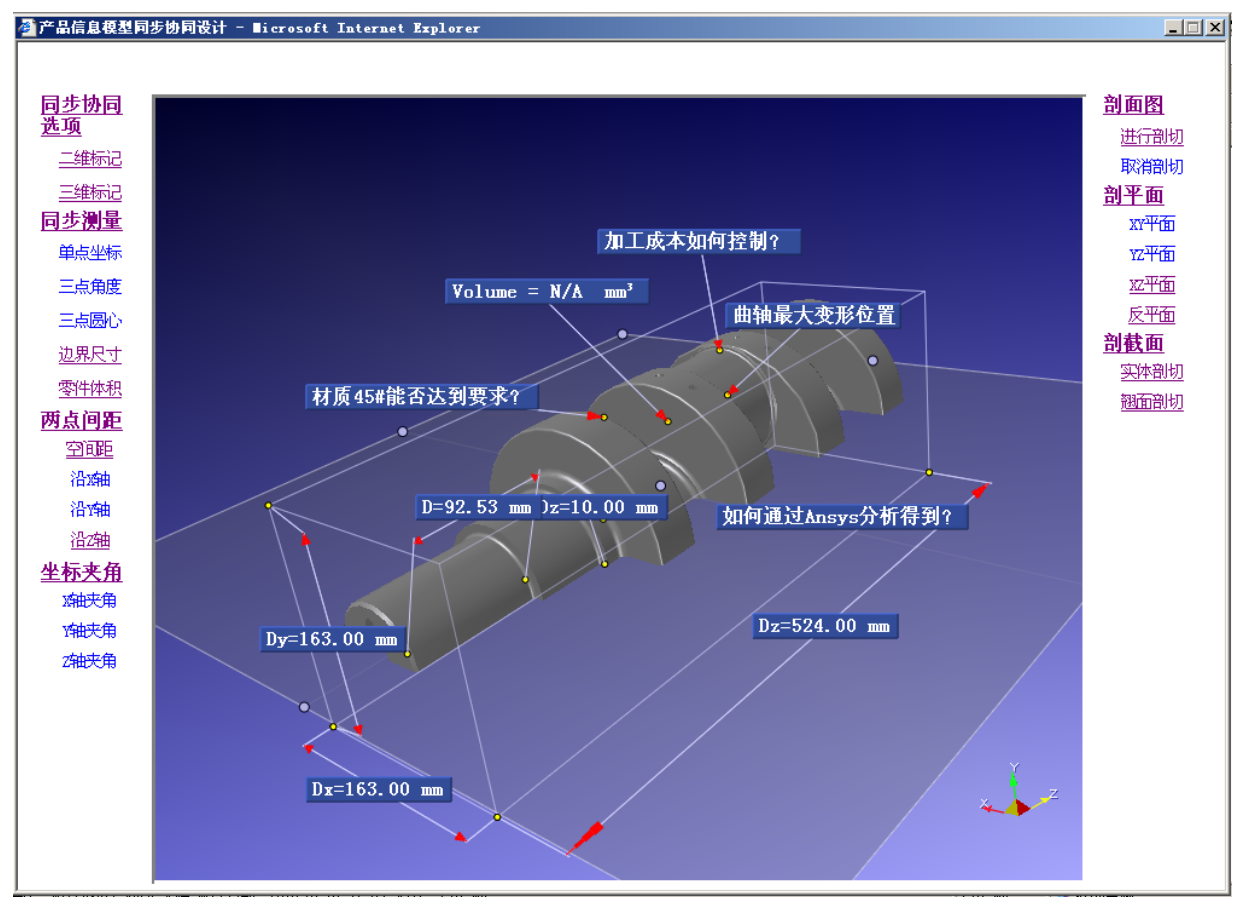

Fig. (4). A sharing CVE for multidisciplinary product design process.

Servlet/Java Bean, Java Applet, etc. Its cross-platform and portability capacity are overall emphasized, and the data management system platform used is an open-source database management system-MySQL. So the collaborative system platform also includes an assistant development environment J2SDK and a runtime environment JRE, and so on. Based on the integrated workspace environment, in this section, pumps products and components are taken as a typical use cases to verify design reviews and activities process, and its real-time CVE is also established for process interaction and control, as shown in Fig. (4). Consequently, in order to resolve produce design conflict, a safe and controlled lightweight collaborative virtual workspace environment is implemented.

\section{CONCLUSION}

In this paper, in order to meet the requirements of multidisciplinary domains collaborative product design, a process modeling method and a conflict resolution algorithm are both put forward based on the product information SVM. On the basis of lightweight format conversion mode from a third party middle file, the construction method and release mechanism of the SVM are achieved in the network CVE. Based on the eEPC model, the interactive process model of collaborative design activities is established, and the conflict resolution model of design requests is also proposed for the design activities process. The analysis and discussion of performance and effectiveness of the collaborative process is comprehensively completed. Finally, based on the J2EE architecture, the application and verification work of synthesis solutions presented are put into effect. Outcomes suggest that the method and system are feasible and reasonable in general.

\section{CONFLICT OF INTEREST}

The author confirms that this article content has no conflict of interest.

\section{ACKNOWLEDGEMENTS}

This work was partly supported by the National Natural Science Foundation of China (No.50975183), the National High-Tech. R\&D Program of China (No.2009AA04Z167), the Program for Liaoning Excellent Talents in University (No.LJQ2012019), Shenyang Science and Technology Plan Program (No.F12-258-1-00) and the Program from Liaoning Province Department of Education (No.L2012067).

\section{REFERENCES}

[1] C. Liu, R.S. Alejandro and G. F. Yin, "Customer-driven product design and evaluation method for collaborative design environments", Journal of Intelligent Manufacturing, vol. 22, pp. 751-764, 2011.

[2] D. J. He, X. Song, Q. Wang and C. Xu, "Method for complex product collaborative design based on cloud service", Computer Integrated Manufacturing Systems, vol. 17, no. 3, pp. 533-539, 2011.

[3] M. Sadeghi, K. H. Hamou and F. Noël, "A collaborative platform architecture for coherence management in multi-view integrated product modeling", International Journal of Computer Integrated Manufacturing, vol. 23, no. 3, pp. 270-282, 2010.

[4] C. Kim, J. Lee, K. Kim, J. Lee and K. Ryu, "A collaborative design framework for the Korean automotive parts industry", International Journal of Computer Integrated Manufacturing, vol. 25, no. 11, pp. 1047-1062, 2012.

[5] S. Bettaieb and F. Noël, "A generic architecture to synchronise design models issued from heterogeneous business tools: towards more interoperability between design expertises", Engineering with Computers, vol. 24, no. 1, pp. 27-41, 2008. 
[6] J.S. Lee, H. Chae, C.-H. Kim and K. Kim, "Design of product ontology architecture for collaborative enterprises", Expert Systems with Applications, vol. 36, no. 2, pp. 2300-2309, 2009.

[7] Actify Inc. http://www.actify.com [Accessed: Jun. 8, 2014]

[8] P. F. Zeng, Y. P. Hao, Y.X. Liu and W.P. Shao, "Product collaborative design method based-on a sharing information model", Journal of Advanced Manufacturing Systems, vol. 10, no. 1, pp. 191198, 2011.
[9] B. Sari, T. Sen and S. E. Kilic, "Ahp model for the selection of partner companies in virtual enterprises", International Journal of Advanced Manufacturing Technology, vol. 38, pp. 367-376, 2008.

[10] P. F. Zeng, Y.X. Liu, Y.P. Hao and G.Q. Sun, "Design model sharing and interactive technique in multidisciplinary collaborative process", Journal of Northeastern University(Natural Science), vol. 30, no. 3, pp. 426-429, 2009.

Received: November 14, 2014

(C) Zeng et al.; Licensee Bentham Open.

This is an open access article licensed under the terms of the Creative Commons Attribution Non-Commercial License (http://creativecommons.org/licenses/by-nc/3.0/) which permits unrestricted, non-commercial use, distribution and reproduction in any medium, provided the work is properly cited. 